\title{
Constitutive formulation of strain-rate sensitive deformation in orthotropic composites
}

\author{
K. Micallef ${ }^{1}$, A. Soleiman-Fallah ${ }^{1}$, P. T. Curtis ${ }^{2}$, D. J. Pope ${ }^{2}$ \\ \& L. A. Louca ${ }^{1}$ \\ ${ }^{I}$ Department of Civil Engineering, Imperial College, London, UK \\ ${ }^{2}$ Physical Sciences Department, DSTL Porton Down, UK
}

\begin{abstract}
The aim of the current study is to develop a constitutive model which captures the full orthotropic behaviour of a laminated composite by employing 9 material parameters and also taking into account strain-rate sensitivity to loading.

The formulation is an extension of the work by Ogihara and Reifsnider (DOI: 10.1023/A:1016069220255), whose model considers 4 parameters, and with the inclusion of strain-rate effect considerations using the method employed by Thiruppukuzhi and Sun (DOI: 10.1016/S0266-3538(00)00133-0).

A plastic potential function which can describe plasticity in all directions, including fibre plasticity, is chosen and using an associated flow rule, the plastic strain-rate components are derived. The plastic compliance matrix is assembled, using a rate-dependent visco-plastic modulus. The elastic compliance matrix is combined with its plastic counterpart to give a rate-form constitutive law.

It is found that the proposed model accounts for strain-rate dependence and by correct choice of model parameters, the model can also be used for various composite architectures, including woven and uni-directional architectures.
\end{abstract}

Keywords: Composites, orthotropic, constitutive modelling, strain-rate effects.

\section{Introduction}

The formulation of a comprehensive constitutive model is imperative for the proper understanding of a material's behaviour under different loading conditions. This includes fibre-reinforced laminated polymeric composites. For efficient use of composite materials under extreme loading, it is necessary to 
consider their response beyond the linear-elastic regime and for different loading rates.

Composites are, in general, non-isotropic materials and tend to exhibit highly non-linear behaviour under various kinds of dynamic loadings. The severe loading nature of dynamic loads also implies that strain-rate effects have a repercussion on the stress-strain response of the material. In addition, it is common for a material to be loaded beyond its elastic limit and deform plastically, giving rise to plastic strains which lead to progressive damage of the material.

Thus, it is necessary to develop a constitutive relationship which can predict non-linear, large strain, strain-rate dependent behaviour to failure of anisotropic uni-directional and/or woven composites which are subjected to dynamic loads.

When a material, including a composite, is subjected to severe loads, it is subjected to significantly high stresses and strains. Thus, when one analyses a composite structure using a linear elastic technique and using a conventional failure criterion to describe failure at a ply level, one would find that failure is attained immediately and throughout the structure. Designing a structure not to fail and to remain elastic under high loads will make the structures thicker and thus heavier and more expensive.

Thus, it is required to have a non-linear response which can describe the material performance beyond the first ply failure and which can model plastic damage sustained by the composite when loaded at high strain-rates.

\subsection{Constitutive models for composite materials}

The constitutive model of a material can be defined as a relation used to characterise its physical properties and such a model will be used to describe the material response of the material when subjected to loading.

The non-linear response of composites has long been recognised to be an important consideration in constitutive modelling. It is common in non-linear constitutive theory to develop the concept of effective stress, $\bar{\sigma}$, and effective plastic strain, $\bar{\varepsilon}^{p}$. This is similar to classical plasticity theory in metals, where the non-linearity is defined on the basis of the effective quantities only.

In 1973, Hahn and Tsai [1] used a complementary elastic energy density function to derive a stress-strain relation which is linear in the longitudinal and transverse directions but non-linear in the in-plane shear. The non-linearity is taken into account by the inclusion of a bi-quadratic term.

A non-linear plastic theory was first applied to composites by Sun and Chen [2], who suggested that a plastic potential function could be applied to unidirectional composites. Using a simple one parameter plastic flow rule, the potential function adopted was essentially a derivative of Hill's [3] generalised plastic potential for a 3D orthotropic material. Sun and Chen, however, assumed a plane stress scenario, imposing that there is no plastic strain in the fibre direction and limiting plasticity only to the matrix.

This assumption is unacceptable in the case of woven composites, since experimental data [4] has shown that non-linearity did occur when material was loaded in the $0^{\circ}$ and $90^{\circ}$ directions. 
In fact, Ogihara and Reifsnider [4] expanded the work of Sun and Chen by using a more general plastic potential with four unknown parameters. These parameters were determined by a number of simple tension experiments for different specimen angles. The effective stress and effective plastic strain were found for each test angle and the parameter combinations resulted in all effective stress-effective plastic strain curves to converge into a single master curve.

The concept of using a master curve has been shown to be valid for various materials by Sun, both in his original 1989 work [2] and also in subsequent works [5-8]. Non-linearity is expressed by a function representing the master curve. Sun and Chen [2] had proposed a power law relating effective stress to effective strain:

$$
\bar{\varepsilon}^{p}=A \bar{\sigma}^{n}
$$

Weeks and Sun [6] found that the exponent $n$ in the above relation was appropriate at all tested strain-rates and thus rate independent. Thus, the rate sensitivity is described solely by the parameter $A$.

For composites to be used under dynamic loading, the effects of strain-rate need to be included in the constitutive model. Thiruppukuzhi and Sun $[5,7]$ proposed a developed power law describing the strain-rate dependence of $A$ :

$$
A=\chi\left(\overline{\dot{\varepsilon}}^{p}\right)^{m}
$$

Thus:

$$
\bar{\varepsilon}^{p}=\chi\left(\overline{\bar{\varepsilon}}^{p}\right)^{m} \bar{\sigma}^{n}
$$

More recently, Hufner and Accorsi [9] have extended the four parameter plastic potential function of Ogihara and Reifsnider to include strain-rate effects using the strain-dependent power law of Thiruppukuzhi and Sun described above.

It should be noted that the non-linear behaviour is characterised on a macroscopic level. Although it is desirable to predict non-linear response of woven composites on a micromechanical scale, i.e., based on the properties of the fibres and the matrix, many workers have shown that it is difficult to achieve this, even for uni-directional composites [4].

\subsection{Choice and method of formulation}

The present work will extend the formulation of Ogihara and Reifsnider from a model with 4 parameters to consider all possible parameters (i.e., 9) and taking into account the strain-rate effects as in the work of Thiruppukuzhi and Sun. 


\section{Derivation of a rate-dependent constitutive model}

In the present study, a plastic potential function which has the most general form with 9 unknown parameters, that could be applied to both anisotropic unidirectional and woven composites is proposed, namely:

$$
\begin{gathered}
2 f\left(\sigma_{i j}\right)=a_{11} \sigma_{11}{ }^{2}+a_{22}{\sigma_{22}}^{2}+a_{33} \sigma_{33}{ }^{2}+2 a_{44} \sigma_{23}{ }^{2}+ \\
2 a_{55}{\sigma_{13}}^{2}+2 a_{66} \sigma_{12}{ }^{2}+2 a_{12} \sigma_{11} \sigma_{22}+2 a_{13} \sigma_{11} \sigma_{33}+ \\
2 a_{23} \sigma_{22} \sigma_{33}
\end{gathered}
$$

Using appropriate values for the various parameters, the function could be used to describe a range of material systems. For example, choosing $a_{11}=$ $a_{12}=0$ and $a_{22}=1$ reduces the above potential function to the one parameter potential of Sun and Chen [2] for uni-directional composites. Similarly, by setting $a_{11}=a_{12}=a_{22}=0$ gives the function used by Thiruppukuzhi and Sun [7] for woven composites.

The generalised anisotropic constitutive equations, in rate form, are expressed as follows:

$$
[\dot{\varepsilon}]=[C][\dot{\boldsymbol{\sigma}}]
$$

In expanded form, this is written as:

$$
\left\{\begin{array}{l}
\dot{\varepsilon}_{11} \\
\dot{\varepsilon}_{22} \\
\dot{\varepsilon}_{33} \\
\dot{\varepsilon}_{23} \\
\dot{\varepsilon}_{13} \\
\dot{\varepsilon}_{12}
\end{array}\right\}=\left[\begin{array}{llllll}
C_{11} & C_{12} & C_{13} & C_{14} & C_{15} & C_{16} \\
C_{21} & C_{22} & C_{23} & C_{24} & C_{25} & C_{26} \\
C_{31} & C_{32} & C_{33} & C_{34} & C_{35} & C_{36} \\
C_{41} & C_{42} & C_{43} & C_{44} & C_{45} & C_{46} \\
C_{51} & C_{52} & C_{53} & C_{54} & C_{55} & C_{56} \\
C_{61} & C_{62} & C_{63} & C_{64} & C_{65} & C_{66}
\end{array}\right]\left\{\begin{array}{c}
\dot{\sigma}_{11} \\
\dot{\sigma}_{22} \\
\dot{\sigma}_{33} \\
\dot{\sigma}_{23} \\
\dot{\sigma}_{13} \\
\dot{\sigma}_{12}
\end{array}\right\}
$$

The strain-rate tensor is decomposed into two components, namely the elastic and the plastic strain-rate components:

$$
\varepsilon_{i j}=\varepsilon_{i j}^{e}+\varepsilon_{i j}^{p}
$$

Thus, the compliance matrix is expressed as the sum of the elastic and plastic components:

$$
[\dot{\varepsilon}]=[C][\dot{\sigma}]=\left[C^{e}+C^{p}\right][\dot{\sigma}]
$$

Each part of the compliance matrix will be derived in turn in the following sections. 


\subsection{Elastic compliance matrix}

The elastic part of the compliance matrix is the standard orthotropic compliance relation obtained from standard textbooks, such as Jones [10]:

$$
\left[\boldsymbol{C}^{\boldsymbol{e}}\right]=\left[\begin{array}{cccccc}
\frac{1}{E_{1}} & -\frac{v_{21}}{E_{2}} & -\frac{v_{31}}{E_{3}} & 0 & 0 & 0 \\
-\frac{v_{12}}{E_{1}} & \frac{1}{E_{2}} & -\frac{v_{32}}{E_{3}} & 0 & 0 & 0 \\
-\frac{v_{13}}{E_{1}} & -\frac{v_{23}}{E_{2}} & \frac{1}{E_{3}} & 0 & 0 & 0 \\
0 & 0 & 0 & \frac{1}{G_{23}} & 0 & 0 \\
0 & 0 & 0 & 0 & \frac{1}{G_{31}} & 0 \\
0 & 0 & 0 & 0 & 0 & \frac{1}{G_{12}}
\end{array}\right]
$$

This matrix is symmetric, i.e. $C_{i j}^{e}=C_{j i}^{e}$ and has effectively only nine unequal constants since:

$$
\frac{v_{i j}}{E_{i}}=\frac{v_{j i}}{E_{j}}
$$

The elastic constants in this matrix are assumed to be rate-dependent in the work of Hufner and Accorsi [9] but Thiruppukuzhi and Sun [7] state that there is no strain-rate dependence in the elastic part of loading. This is assumed to be a valid simplification for the purpose of this study.

\subsection{Plastic compliance matrix}

The plastic part of the compliance matrix is assembled by first computing the plastic strain-rate components from the potential function using an associated flow rule:

$$
\dot{\varepsilon}_{i j}^{p}=\dot{\lambda} \frac{\partial f\left(\sigma_{i j}\right)}{\partial \sigma_{i j}}
$$

where $\dot{\lambda}$ is the proportionality factor rate.

Applying this flow rule to the chosen plastic potential, the plastic strain-rate components are: 


$$
\begin{gathered}
\dot{\varepsilon}_{11}^{p}=\dot{\lambda}\left(a_{11} \sigma_{11}+a_{12} \sigma_{22}+a_{13} \sigma_{33}\right) \\
\dot{\varepsilon}_{22}^{p}=\dot{\lambda}\left(a_{22} \sigma_{22}+a_{23} \sigma_{33}+a_{12} \sigma_{11}\right) \\
\dot{\varepsilon}_{33}^{p}=\dot{\lambda}\left(a_{33} \sigma_{33}+a_{23} \sigma_{22}+a_{13} \sigma_{11}\right) \\
\dot{\varepsilon}_{23}^{p}=\dot{\lambda}\left(2 a_{44} \sigma_{23}\right) \\
\dot{\varepsilon}_{13}^{p}=\dot{\lambda}\left(2 a_{55} \sigma_{13}\right) \\
\dot{\varepsilon}_{12}^{p}=\dot{\lambda}\left(2 a_{66} \sigma_{12}\right)
\end{gathered}
$$

The proportionality factor rate, $\dot{\lambda}$, is derived using the equivalence of the rate of plastic work, $\dot{W}^{p}$, namely:

$$
\dot{W}^{p}=\bar{\sigma} \overline{\dot{\varepsilon}}^{p}=\sigma_{i j} \dot{\varepsilon}_{i j}^{p}
$$

Rearranging the equation for the flow rule:

$$
\dot{\lambda}=\frac{\bar{\sigma} \bar{\varepsilon}^{p}}{2 f}
$$

From classical plasticity theory, $\bar{\sigma}=\sqrt{3 f}$ and thus:

$$
f=\frac{\bar{\sigma}^{2}}{3}
$$

This implies that $\dot{\lambda}$ can be expressed as a function of $\overline{\bar{\varepsilon}}^{p}$ and $\bar{\sigma}$ only:

$$
\dot{\lambda}=\frac{\bar{\sigma} \bar{\varepsilon}^{p}}{2 f}=\frac{3 \bar{\sigma} \overline{\dot{\varepsilon}}^{p}}{2 \bar{\sigma}^{2}}=\frac{3 \bar{\varepsilon}^{p}}{2 \bar{\sigma}}
$$

A rate-dependent visco-plastic modulus, $H_{p}$, is defined as:

$$
H_{p}=\frac{\dot{\bar{\sigma}}}{\overline{\bar{\varepsilon}}^{p}}=\frac{d \bar{\sigma}}{d \bar{\varepsilon}^{p}}
$$

Thus, the proportionality factor rate is expressed as:

$$
\dot{\lambda}=\frac{3 \dot{\bar{\sigma}}}{2 H_{p} \bar{\sigma}}
$$

Using the definition of $\bar{\varepsilon}^{p}$ proposed by Thiruppukuzhi and Sun [5,7] given in eqn (3) earlier and differentiating it with respect to $\bar{\sigma}$,

$$
\frac{d \bar{\varepsilon}^{p}}{d \bar{\sigma}}=n \chi\left(\bar{\varepsilon}^{p}\right)^{m} \bar{\sigma}^{n-1}
$$


This implies that:

$$
H_{p}=\frac{d \bar{\sigma}}{d \bar{\varepsilon}^{p}}=\frac{1}{n \chi\left(\overline{\dot{\varepsilon}}^{p}\right)^{m} \bar{\sigma}^{n-1}}
$$

The effective stress-rate is given as:

$$
\begin{aligned}
\dot{\bar{\sigma}}=\frac{3}{2 \bar{\sigma}} & {\left[\left(a_{11} \sigma_{11}+a_{12} \sigma_{22}+a_{13} \sigma_{33}\right) \dot{\sigma}_{11}\right.} \\
& +\left(a_{22} \sigma_{22}+a_{23} \sigma_{33}+a_{12} \sigma_{11}\right) \dot{\sigma}_{22} \\
& +\left(a_{33} \sigma_{33}+a_{23} \sigma_{22}+a_{13} \sigma_{11}\right) \dot{\sigma}_{33} \\
& +\left(2 a_{44} \sigma_{23}\right) \dot{\sigma}_{12}+\left(2 a_{55} \sigma_{13}\right) \dot{\sigma}_{13} \\
& \left.+\left(2 a_{66} \sigma_{12}\right) \dot{\sigma}_{23}\right]
\end{aligned}
$$

Finally, after substitution, the proportionality factor rate is given by:

$$
\begin{aligned}
\dot{\lambda}=\frac{3 \dot{\bar{\sigma}}}{2 H_{p} \bar{\sigma}} & =\frac{3}{2 H_{p} \bar{\sigma}}\left\{\frac { 3 } { 2 \overline { \sigma } } \left[\left(a_{11} \sigma_{11}+a_{12} \sigma_{22}\right.\right.\right. \\
& \left.+a_{13} \sigma_{33}\right) \dot{\sigma}_{11} \\
& +\left(a_{22} \sigma_{22}+a_{23} \sigma_{33}+a_{12} \sigma_{11}\right) \dot{\sigma}_{22} \\
& +\left(a_{33} \sigma_{33}+a_{23} \sigma_{22}+a_{13} \sigma_{11}\right) \dot{\sigma}_{33} \\
& +\left(2 a_{44} \sigma_{23}\right) \dot{\sigma}_{12}+\left(2 a_{55} \sigma_{13}\right) \dot{\sigma}_{13} \\
& \left.\left.+\left(2 a_{66} \sigma_{12}\right) \dot{\sigma}_{23}\right]\right\}
\end{aligned}
$$

Thus:

$$
\begin{aligned}
\dot{\lambda}=\frac{9}{4 H_{p}} & \bar{\sigma}^{2} \\
+ & {\left[\left(a_{11} \sigma_{11}+a_{12} \sigma_{22}+a_{13} \sigma_{33}\right) \dot{\sigma}_{11}\right.} \\
& +\left(a_{22} \sigma_{22}+a_{23} \sigma_{33}+a_{12} \sigma_{11}\right) \dot{\sigma}_{22} \\
& +\left(a_{33} \sigma_{33}+a_{23} \sigma_{22}+a_{13} \sigma_{11}\right) \dot{\sigma}_{33} \\
& +\left(2 a_{44} \sigma_{23}\right) \dot{\sigma}_{12}+\left(2 a_{55} \sigma_{13}\right) \dot{\sigma}_{13} \\
& \left.+\left(2 a_{66} \sigma_{12}\right) \dot{\sigma}_{23}\right]
\end{aligned}
$$

By substituting this expression for $\dot{\lambda}$ into the plastic strain-rate components derived earlier in eqn (12), it is possible to find the individual terms of the plastic compliance matrix.

The derivation requires extensive algebraic manipulation and, for the sake of brevity, only one full derivation of the first row of the plastic compliance matrix $\left(\dot{\varepsilon}_{1 i}^{p}, i=1-6\right)$ is presented as an example. 
404 Computational Methods and Experimental Measurements XV

$$
\begin{aligned}
\dot{\varepsilon}_{11}^{p}= & \dot{\lambda}\left(a_{11} \sigma_{11}+a_{12} \sigma_{22}+a_{13} \sigma_{33}\right) \\
& =\left\{\frac { 9 } { 4 H _ { p } \overline { \sigma } ^ { 2 } } \left[\left(a_{11} \sigma_{11}+a_{12} \sigma_{22}\right.\right.\right. \\
& \left.+a_{13} \sigma_{33}\right) \dot{\sigma}_{11} \\
& +\left(a_{22} \sigma_{22}+a_{23} \sigma_{33}+a_{12} \sigma_{11}\right) \dot{\sigma}_{22} \\
& +\left(a_{33} \sigma_{33}+a_{23} \sigma_{22}+a_{13} \sigma_{11}\right) \dot{\sigma}_{33} \\
& +\left(2 a_{44} \sigma_{23}\right) \dot{\sigma}_{12}+\left(2 a_{55} \sigma_{13}\right) \dot{\sigma}_{13} \\
& \left.\left.+\left(2 a_{66} \sigma_{12}\right) \dot{\sigma}_{23}\right]\right\}\left(a_{11} \sigma_{11}+a_{12} \sigma_{22}\right. \\
& \left.+a_{13} \sigma_{33}\right)
\end{aligned}
$$

Upon expansion and simplification,

$$
\begin{aligned}
\dot{\varepsilon}_{11}^{p}=\frac{9}{4 H_{p} \bar{\sigma}^{2}}[ & \left(a_{11}^{2} \sigma_{11}^{2}+2 a_{11} a_{12} \sigma_{11} \sigma_{22}+2 a_{11} a_{13} \sigma_{11} \sigma_{33}\right. \\
& \left.+2 a_{12} a_{13} \sigma_{22} \sigma_{33}+a_{12}^{2} \sigma_{22}^{2}+a_{13}^{2} \sigma_{33}^{2}\right) \dot{\sigma}_{11} \\
& +\left(a_{11} a_{12} \sigma_{11}^{2}+a_{22} a_{12} \sigma_{22}^{2}+a_{13} a_{23} \sigma_{33}^{2}\right. \\
& +a_{11} a_{22} \sigma_{11} \sigma_{22} \\
& +a_{11} a_{23} \sigma_{11} \sigma_{33}+a_{12} a_{13} \sigma_{11} \sigma_{33} \\
& +a_{12} a_{23} \sigma_{22} \sigma_{33}+a_{13} a_{22} \sigma_{22} \sigma_{33} \\
& \left.+a_{12}^{2} \sigma_{22} \sigma_{33}\right) \dot{\sigma}_{22} \\
& +\left(a_{11} a_{13} \sigma_{11}^{2}+a_{23} a_{12} \sigma_{22}^{2}+a_{13} a_{33} \sigma_{33}^{2}\right. \\
& +a_{11} a_{23} \sigma_{11} \sigma_{22} \\
& +a_{12} a_{13} \sigma_{11} \sigma_{22}+a_{11} a_{13} \sigma_{11} \sigma_{33} \\
& +a_{12} a_{33} \sigma_{22} \sigma_{33}+a_{13} a_{23} \sigma_{22} \sigma_{33} \\
& \left.+a_{13}^{2} \sigma_{11} \sigma_{33}\right) \dot{\sigma}_{33} \\
& +2\left(a_{11} a_{44} \sigma_{11} \sigma_{23}+a_{12} a_{44} \sigma_{22} \sigma_{23}\right. \\
& \left.+a_{13} a_{44} \sigma_{33} \sigma_{23}\right) \dot{\sigma}_{23} \\
& +2\left(a_{11} a_{55} \sigma_{11} \sigma_{13}+a_{12} a_{55} \sigma_{22} \sigma_{13}\right. \\
& \left.+a_{13} a_{55} \sigma_{33} \sigma_{13}\right) \dot{\sigma}_{13}+2\left(a_{11} a_{66} \sigma_{11} \sigma_{12}\right. \\
& \left.\left.+a_{12} a_{66} \sigma_{22} \sigma_{12}+a_{13} a_{66} \sigma_{33} \sigma_{12}\right) \dot{\sigma}_{12}\right]
\end{aligned}
$$

Extracting the co-efficients of each $\dot{\sigma}_{i j}$ gives the entries for the plastic compliance matrix. For example, the first entry of the first row of the compliance matrix would be:

$$
\begin{gathered}
C_{11}^{p}=\frac{9}{4 H_{p} \bar{\sigma}^{2}}\left(a_{11}^{2} \sigma_{11}^{2}+2 a_{11} a_{12} \sigma_{11} \sigma_{22}+2 a_{11} a_{13} \sigma_{11} \sigma_{33}\right. \\
\left.+2 a_{12} a_{13} \sigma_{22} \sigma_{33}+a_{12}^{2} \sigma_{22}^{2}+a_{13}^{2} \sigma_{33}^{2}\right)
\end{gathered}
$$

A similar procedure yields the rest of the terms in the plastic compliance matrix, which are presented in Appendix 1. It is noted that the plastic compliance matrix is also symmetric, i.e. $C_{i j}^{p}=C_{j i}^{p}$ and thus the elasto-plastic compliance matrix is overall symmetric. 


\section{Conclusions and future work}

The framework for a constitutive model which captures the full orthotropic behaviour of a laminated composite has been developed. This model employs 9 material parameters and also takes into account strain-rate sensitivity to loading. Thus, the rate form constitutive relationship is defined in terms of a compliance matrix which also includes a visco-plastic modulus.

The devised model will be implemented in a user-defined material subroutine (VUSDFLD and/or VUMAT) in the finite element code ABAQUS/Explicit, using material properties obtained from small-specimen basic tensile, compressive and shear tests at different strain-rates on specimens of various composite layups under dynamic loads.

The proposed constitutive model will also be validated using larger $300 \times 300 \mathrm{~mm}$ panels of the same composites tested earlier. A series of parametric studies will be performed using the numerical model to assess the overall response sensitivity of the proposed material model to the parameters defining it uniquely, such as modulii, Poisson ratios or yield locus. The study will include both small specimens as well as large panels.

\section{Acknowledgements}

This work is part of a research project jointly funded by the Defence Science and Technology Laboratory (DSTL) and the Engineering and Physical Sciences Research Council (EPSRC).

\section{Appendix}

The complete list of the elasto-plastic compliance matrix terms are given below:

$$
\begin{aligned}
& C_{11}=\frac{1}{E_{1}}+\frac{9}{4 H_{p} \bar{\sigma}^{2}}\left(a_{11}^{2} \sigma_{11}^{2}+2 a_{11} a_{12} \sigma_{11} \sigma_{22}+2 a_{11} a_{13} \sigma_{11} \sigma_{33}\right. \\
& \left.+2 a_{12} a_{13} \sigma_{22} \sigma_{33}+a_{12}^{2} \sigma_{22}^{2}+a_{13}^{2} \sigma_{33}^{2}\right)
\end{aligned}
$$




$$
\begin{gathered}
C_{13}=C_{31}=-\frac{v_{31}}{E_{3}} \\
+\frac{9}{4 H_{p} \bar{\sigma}^{2}}\left(a_{11} a_{13} \sigma_{11}^{2}+a_{23} a_{12} \sigma_{22}^{2}+a_{13} a_{33} \sigma_{33}^{2}\right. \\
+a_{11} a_{23} \sigma_{11} \sigma_{22} \\
+a_{12} a_{13} \sigma_{11} \sigma_{22}+a_{11} a_{13} \sigma_{11} \sigma_{33}+a_{12} a_{33} \sigma_{22} \sigma_{33} \\
\left.+a_{13} a_{23} \sigma_{22} \sigma_{33}+a_{13}^{2} \sigma_{11} \sigma_{33}\right)
\end{gathered}
$$




$$
\begin{gathered}
C_{35}=C_{53}=\frac{9 a_{55}}{2 H_{p} \bar{\sigma}^{2}}\left(a_{13} \sigma_{11} \sigma_{13}+a_{23} \sigma_{22} \sigma_{13}+a_{33} \sigma_{33} \sigma_{13}\right) \\
C_{36}=C_{63}=\frac{9 a_{66}}{2 H_{p} \bar{\sigma}^{2}}\left(a_{13} \sigma_{11} \sigma_{12}+a_{23} \sigma_{22} \sigma_{12}+a_{33} \sigma_{33} \sigma_{12}\right) \\
C_{44}=\frac{1}{G_{23}}+\frac{9 a_{44}^{2} \sigma_{23}^{2}}{H_{p} \bar{\sigma}^{2}} \\
C_{45}=C_{54}=\frac{9 a_{44} a_{55} \sigma_{23} \sigma_{13}}{H_{p} \bar{\sigma}^{2}} \\
C_{46}=C_{64}=\frac{9 a_{44} a_{66} \sigma_{23} \sigma_{12}}{H_{p} \bar{\sigma}^{2}} \\
C_{55}=\frac{1}{G_{31}}+\frac{9 a_{55}^{2} \sigma_{13}^{2}}{H_{p} \bar{\sigma}^{2}} \\
C_{56}=C_{65}=\frac{9 a_{55} a_{66} \sigma_{13} \sigma_{12}}{H_{p} \bar{\sigma}^{2}} \\
C_{66}=\frac{1}{G_{12}}+\frac{9 a_{66}^{2} \sigma_{12}^{2}}{H_{p} \bar{\sigma}^{2}}
\end{gathered}
$$

\section{References}

[1] Hahn, H.T. and S.W. Tsai, Nonlinear elastic behaviour of unidirectional composite laminae. Journal of Composite Materials, 1973. 7(1): p. 102-118.

[2] Sun, C.T. and J.L. Chen, A simple flow rule for characterizing nonlinear behaviour of fibre composites. Composites, 1989. 23(10): p. 1009-1020.

[3] Hill, R., A theory of the yielding and plastic flow of anisotropic metals. Proceedings of the Royal Society of London. Series A. Mathematical and Physical Sciences, 1948. 193(1033): p. 281-297.

[4] Ogihara, S. and K. L. Reifsnider, Characterization of nonlinear behavior in woven composite laminates. Applied composite materials, 2002. 9(4): p. 249.

[5] Thiruppukuzhi, S.V. and C.T. Sun, Testing and modeling high strain rate behavior of polymeric composites. Composites Part B: Engineering, 1998. 29(5): p. 535-546.

[6] Weeks, C.A. and C.T. Sun, Modeling non-linear rate-dependent behavior in fiber-reinforced composites. Composites Science and Technology, 1998. 58(3-4): p. 603-611. 
[7] Thiruppukuzhi, S.V. and C.T. Sun, Models for the strain-rate-dependent behavior of polymer composites. Composites Science and Technology, 2001. 61(1): p. 1-12.

[8] Tsai, J. and C.T. Sun, Constitutive model for high strain rate response of polymeric composites. Composites Science and Technology, 2002. 62(1011): p. 1289-1297.

[9] Hufner, D.R. and M.L. Accorsi, A progressive failure theory for woven polymer-based composites subjected to dynamic loading. Composite Structures, 2009. 89(2): p. 177-185.

[10] Jones, R., Mechanics of composite materials. 1999: Taylor \& Francis Inc. 